\title{
Le taux de conversion de volumes de bois frais en biomasse : amélioration de ses méthodes d'estimation
}

\author{
Jean-Luc BISCH \\ INRA, Station de Sylviculture \\ Centre de Recherches d'Orléans, Ardon, F 45160 Olivet
}

\begin{abstract}
Résumé
On démontre que le principe d'Archimède est utilisé de manière incorrecte pour la détermination du volume d'un échantillon de bois frais. Le fait que l'on néglige le volume d'eau absorbée par celui-ci pendant l'immersion entraîne une sous estimation systématique du volume.

Une formule exacte est établie par une démonstration théorique, puis vérifiée expérimentalement avec des rondelles de chênes non écorcées.

Pour ces rondelles, on supprime ainsi un biais de 1,2 p. 100 en moyenne et le taux de conversion du volume en biomasse défini par le rapport du poids anhydre d'un échantillon à son volume frais est déterminé avec une erreur relative maximale de 0,6 p. 100.

La même précision est obtenue par la méthode du xylomètre, en tenant compte de l'eau absorbée par l'échantillon.

On montre en outre qu'il existe une corrélation entre le volume d'eau absorbée par l'échantillon et le volume de son écorce.
\end{abstract}

Mots clés : Biomasse, méthode d'estimation, échantillon, volume, précision, Quercus.

\section{Introduction}

Avec les perspectives actuelles d'une raréfaction des stocks énergétiques classiques et de l'augmentation de leurs couts d'exploitation, on assiste à une diversification de l'utilisation du bois. Il est employé soit comme matériạu, soit comme matière industrielle pour l'industrie du papier, soit pour la production d'énergie par combustion ou après transformation chimique.

Cette diversification va de pair avec une utilisation complète de l'arbre entier : racines, tronc, branches et feuilles (PARDE, 1977).

C'est dans le cadre d'une utilisation accrue à des fins énergétiques que le besoin s'est fait sentir d'évaluer le potentiel énergétique disponible dans les forêts et que de nombreuses études sur la biomasse forestière ont été entreprises. Citons les travaux de Kestemont (1970, 1971), Auclair \& Metayer (1980), Ranger et al. (1981), Alemdag (1982), Auclair \& Bige (1984), Bouchon et al., (1985). 
La méthodologie d'estimation de la biomasse est maintenant bien connue. La biomasse de l'arbre peut être évaluée par deux méthodes différentes.

L'une consiste à multiplier le rapport poids anhydre/poids frais d'un échantillon convenablement choisi par le poids frais de l'ensemble dont il est représentatif (arbre entier, branche, billon, etc.).

L'autre consiste à appliquer le taux de conversion du volume en biomasse défini par le rapport poids anhydre/volume frais d'un échantillon au volume total de bois dont on veut connaître la valeur en biomasse.

Le poids anhydre est le poids de l'échantillon après passage à l'étuve à $105^{\circ} \mathrm{C}$ jusqu'au poids constant. Nous appelons volume frais d'un échantillon son volume au moment de son prélèvement.

Alors que la première méthode est employée lorsque l'on peut peser à l'état frais tout le bois dont on veut connaître la biomasse (brins de taillis par exemple) et ne présente pas de difficultés particulières, la seconde l'est pour des volumes plus importants de bois dont la pesée n'est pas possible avec une simple balance de terrain.

Cette seconde méthode offre l'inconvénient du passage obligé par la détermination du volume de l'échantillon. C'est à ce problème que nous nous attachons dans cet article.

Le volume frais des échantillons peut être estimé de deux façons différentes.

\subsection{La méthode du xylomètre}

Leguay (1982) l'utilise pour estimer le volume de rondelles prélevées sur des grumes de chênes de taillis sous futaie.

Martin (1984) l'emploie pour estimer le volume de billons de Prunus serotina, de Nyssa sylvatica, et de Quercus sp., ce volume servant de référence à la comparaison entre des volumes obtenus par application de différentes formules de cubage géométrique.

Elle consiste à plonger l'échantillon dans une cuve remplie d'eau et à mesurer indirectement le volume d'eau déplacé, soit par lecture du déplacement du niveau, soit par récupération du volume d'eau déplacé qui est ensuite pesé.

Nous avons constaté que cette méthode était peu satisfaisante pour des rondelles fraîches de chênes non écorcées; en effet, elle ne tient pas compte du volume d'eau absorbé par la rondelle pendant l'immersion.

Martin (1984) constate lui aussi que pour des échantillons dont le volume varie entre 100 et $170 \mathrm{dm}$, le coefficient de variation de ce volume estimé en immergeant 5 fois chacun des échantillons est de l'ordre de 2 à 3 p. 100.

\subsection{Méthode dite "de la poussée d'Archimède»}

Cette méthode, encore appelée méthode hydrostatique, est exposée par CARRE (1972). Il l'utilise pour calculer le volume de rondelles de hêtre, d'épicéa commun et de charme, tant à l'état frais qu'à l'état sec (après passage à l'étuve). 
Elle a notamment été reprise par ScHNock (1983) pour estimer le volume d'échantillons de tiges de charme.

Elle consiste à appliquer le principe de la poussée d'Archimède à la rondelle immergée dans l'eaut.

Son volume frais, supposé égal au volume d'eau déplacé $V$ est tel que :

$$
\mathrm{V} \omega_{0} g=P_{\mathrm{f}}-P_{\mathrm{ap},}
$$

où $Q_{0}$ est la masse volumique de l'eau, $g$ la constante de Newton, $P_{f}$ le poids frais de l'échantillon avant immersion et $P_{a p j}$, son poids apparent dans l'eau.

Or, nous avons constaté que cette formule n'était pas satisfaisante, elle non plus. En effet, il est facile de remarquer qu'au cours de l'immersion, le poids apparent de l'échantillon dans l'eau augmente, souvent rapidement dans les premières secondes, puis de plus en plus lentement. La conséquence directe de ce phénomène est que le volume estimé dépend étroitement du temps d'immersion.

Nous avons démontré en fait que la formule utilisée est abusivement simple et mérite une petite modification.

Cette modification constitue l'objet essentiel de cet article, elle vise à améliorer la précision obtenue sur la valeur du taux de conversion du volume en biomasse calculé par la méthode hydrostatique.

De même, nous verrons qu'il est possible d'améliorer la méthode du xylomètre en tenant compte du volume d'eau absorbé par la rondelle pendant l'immersion.

\section{Etablissement d'une formule correcte pour l'application de la méthode hydrostatique}

Deux démonstrations sont possibles, selon que l'on prend comme système sur lequel s'appliquent les forces, soit la rondelle seule, soit la rondelle plus l'eau qu'elle a absorbée entre le début de l'immersion et l'instant d'équilibre $t$ considéré. Elles aboutissent au même résultat.

Nous ne présentons ici que le raisonnement où le système sur lequel s'appliquent les forces à l'instant d'équilibre $t$ est défini par l'ensemble « rondelle + eau contenue dans la rondelle à l'instant $t »$.

La figure 1 représente schématiquement la méthode de pesée et les forces appliquées au système en équilibre à un instant donné t.

L'équilibre se traduit vectoriellement par l'équation :

$$
\overrightarrow{\mathrm{P}}_{\mathrm{ar}}+\overrightarrow{\mathrm{P}_{\mathrm{r}}}+\overrightarrow{\mathrm{T}}=\overrightarrow{0}
$$

La poussée d'Archimède est par définition égale au poids du volume d'eau déplacé par le système considéré. Le taux d'humidité initial de la rondelle étant supérieur au point de saturation des fibres (taux de 30 p. 100 exprimé par rapport au poids anhydre), on peut admettre qu'elle ne subit pas de déformation dans ce milieu aqueux (Rosen, 1974; VALIı̀̀re, 1975). 


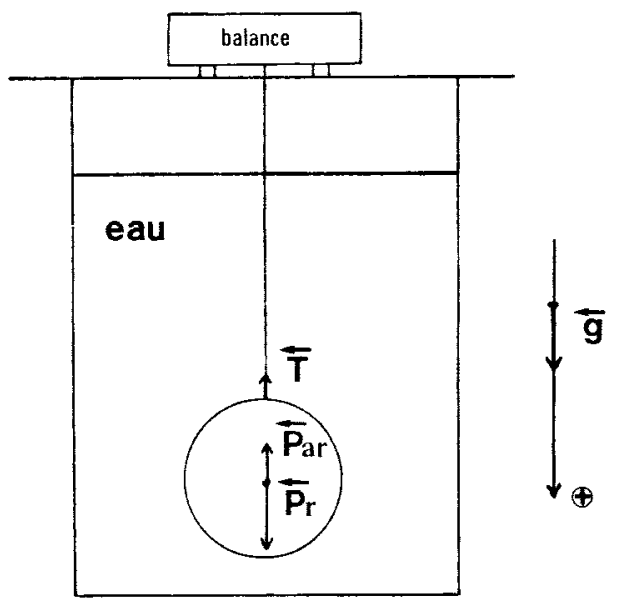

FIG. 1

\section{Représentation schématique de la méthode de pesée}

et des forces appliquées au système «rondelle + eau absorbée » à un instant donné.

Schematic diagram of the weighing method showing the forces applied to the «sample + absorbed water system.

$\overrightarrow{\mathbf{T}}$ : Tension du fil.

Tension of the wire.

$\overrightarrow{\mathrm{P}_{\mathrm{a}}}$ : Poussée d'Archimède.

Archimedean pressure.

$\overrightarrow{\mathbf{P}_{\mathrm{r}}}$ : Poids réel du système.

Real weight of the system.

Le volume d'eau déplacé est égal au volume de la rondelle dans son état frais (noté $\mathrm{V}_{\mathrm{f}}$ ), volume que nous cherchons à connaître.

$\overrightarrow{\mathrm{P}_{\mathrm{ar}}}$ vaut alors $\overrightarrow{\mathrm{P}_{\mathrm{ar}}}=-\mathrm{V}_{\mathrm{f}}{\underline{Q_{0}}}_{\mathrm{g}} \overrightarrow{\mathrm{g}}$

$\overrightarrow{\mathrm{P}_{\mathrm{r}}}$ est le poids réel du système et vaut :

$$
\overrightarrow{\mathrm{P}_{\mathrm{r}}}=\overrightarrow{\mathrm{P}_{\mathrm{f}}}+\mathrm{V}_{\mathrm{o}} Q_{0} \overrightarrow{\mathrm{g}}
$$

où $\vec{P}_{f}$ est le poids frais de la rondelle avant immersion, $V_{0}$ le volume d'eau absorbé par la rondelle entre le début de l'immersion $(t=0)$ et l'instant considéré $t$.

$\vec{T}$ est la tension qu'exerce le fil sur le système, cette force s'oppose au poids apparent du système. $\overrightarrow{\mathrm{T}}=-\overrightarrow{\mathrm{P}}_{\mathrm{ap},}$.

La valeur du poids apparent est lue sur la balance.

L'équation (2) est alors équivalente à :

$$
-\overrightarrow{P_{a p p}}-V_{f} \varrho_{0} \vec{g}+\overrightarrow{P_{f}}+V \overrightarrow{\varrho_{o}} g=0
$$


Par projection sur l'axe vertical orienté positivement selon $\vec{g}$, on obtient algébriquement :

$$
V_{f} \varrho_{0}=P_{f}-P_{a p p}+V_{o} \varrho_{o} g
$$

La comparaison avec l'équation (1) $V_{f} \varrho_{o} g=P_{f}-P_{a p p}$ révèle clairement l'apport du terme $V_{0}, g_{0} g$ qui est le poids du volume d'eau absorbé par la rondelle pendant l'immersion.

Tout calcul ne tenant pas compte de ce terme entraîne une sous-estimation du volume recherché $V_{f}$.

\section{Application expérimentale}

Il s'agit de vérifier si le volume calculé $V_{f}$ est bien indépendant du temps d'immersion et de déterminer la valeur $\mathrm{V}_{0}$ du volume d'eau absorbé.

\subsection{Variation de $P_{a p p}$ en fonction du temps d'immersion}

L'expérience est réalisée 1 à $3 \mathrm{~h}$ après le prélèvement de 9 rondelles sur des grumes de chênes de taillis sous futaie abattus 2 jours plus tôt (hiver 1984-1985). Les chênes, façonnés, n’ont pas pu être identifiés avec certitude; il s'agit de chênes sessiles ou pédonculés.

Le taux d'humidité des rondelles prélevées était en moyenne de 42 p. 100 (par rapport à leur poids frais).

Leurs caractéristiques physiques sont décrites dans le tableau 1. L'échantillonnage couvrant une assez large gamme de dimensions, nous pourrons observer leur influence sur les résultats.

Nous avons observé l'évolution du poids apparent dans l'eau en fonction du temps d'immersion. L'expérience est décrite dans la figure 1. Le chronomètre est déclenché dès que la rondelle entre dans l'eau. Les lectures de poids sont faites toutes les 5 secondes.

On précise que l'on a ajouté aux rondelles une tare qui permettait ainsi leur immersion (elles flottaient). Le poids apparent observé est diminué du poids apparent de la tare; le poids apparent des rondelles est négatif.

Les 9 «courbes» obtenues ont la même allure. Le poids apparent augmente rapidement au début, puis de plus en plus lentement. Cette augmentation correspond à l'augmentation du volume $\mathrm{V}_{0}$ d'eau absorbé par la rondelle. En effet, l'équation (3) est équivalente à :

$$
P_{a p 1}=P_{f}+V_{o} g g-V_{f} \varrho_{o} g
$$

dans laquelle $P_{f}, V_{f}, Q_{0}, g$ sont des constantes.

$Q_{o}$ mesuré à l'aide d'un densitomètre valait $1,001 \pm 0,001 \mathrm{~g} / \mathrm{cm}^{3}$.

Un exemple de courbe obtenue est donné dans la figure 2. Les lectures des poids apparents ont été arrêtées à $4 \mathrm{mn}$ pour deux raisons : d'une part les oscillations 
rapides de la balance au début de l'expérience, liées à l'instabilité de la rondelle dans l'eau, ont disparu; d'autre part la valeur du poids apparent n'augmente plus que très lentement.

C'est cette valeur du poids apparent après $4 \mathrm{mn}$ d'immersion qui sera retenue pour effectuer les calculs à venir.

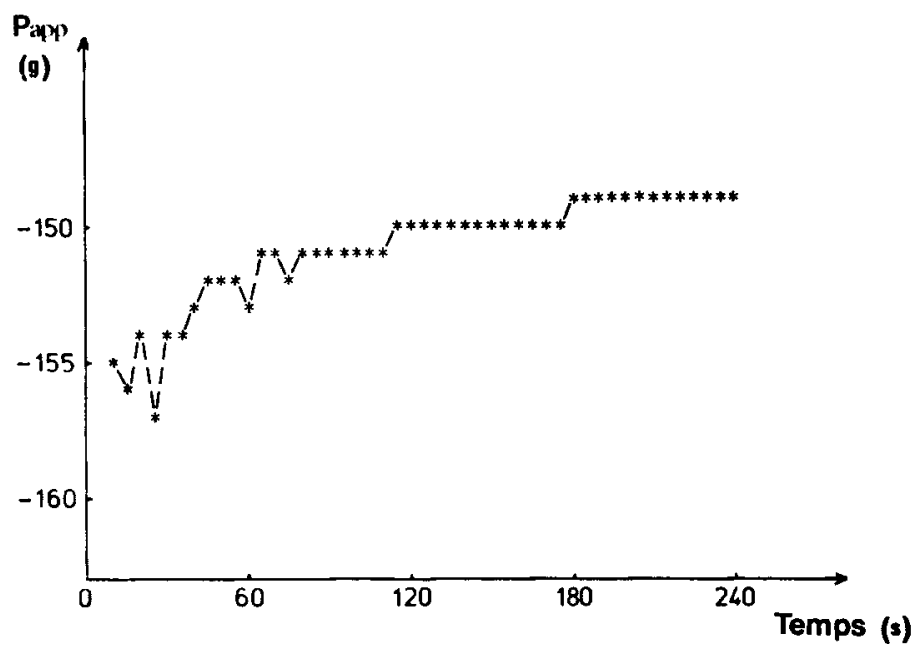

Fig, 2

In/luence de la durée d'immersion sur le poids apparent (rondelle $\left.n^{\circ} 6\right)$.

Influence of immersion time on apparent weight (sample $n^{\circ} 6$ ).

TABLEAU 1

Caractéristiques des 9 rondelles.

Mensurations of the 9 samples.

\begin{tabular}{|c|c|c|c|c|c|c|c|c|c|}
\hline $\mathrm{N}^{\circ}$ rondelle & 1 & 2 & 3 & 4 & 5 & 6 & 7 & 8 & 9 \\
\hline $\mathrm{H}(\mathrm{mm})$ & 95 & 495 & 259 & 349 & 116 & 72 & 226 & 82 & 83 \\
\hline $\mathrm{C}(\mathrm{mm})$ & 733 & 383 & 600 & 476 & 918 & 1162 & 450 & 757 & 769 \\
\hline e $(\mathrm{mm})$ & 15 & 7 & 12 & 9 & 13 & 15 & 9 & 14 & 14 \\
\hline$P_{f}(g)$ & 3723 & 5322 & 6850 & 5920 & 7224 & 7143 & 3403 & 3460 & 3560 \\
\hline
\end{tabular}

H : Hauteur (ou épaisseur moycnne).

Height (or mean thickness).

C : Circonférence au milieu. Girth.

e : Epaisseur d'écorce.

Thickness of the bark.

$P_{f}$ : Poids frais.

Fresh weight. 
Les rondelles sont retirées de l'eau immédiatement après la lecture de cette dernière valeur, et aussitôt utilisées pour la recherche des volumes $V_{0}$ et $V_{f}$.

\subsection{Détermination de $V_{0}$ et de $V_{f}$}

La difficulté réside dans la détermination de $V_{o}$, il n'est en effet pas possible de mesurer directement ce volume; on doit se contenter d'en faire une estimation en fonction du poids de la rondelle sortie de l'eau, lequel diminue dès que la rondelle est émergée.

Le problème est de déterminer au bout de combien de temps il est souhaitable de peser la rondelle pour obtenir une estimation supposée bonne de $V_{0}$ par différence entre le poids après immersion et le poids avant immersion. Pour tenter de le résoudre, nous avons observé l'allure de la courbe représentant le poids de la rondelle en fonction du temps dès qu'elle est retirée de l'eau.

Le système de pesée est schématisé par la figure 3 .

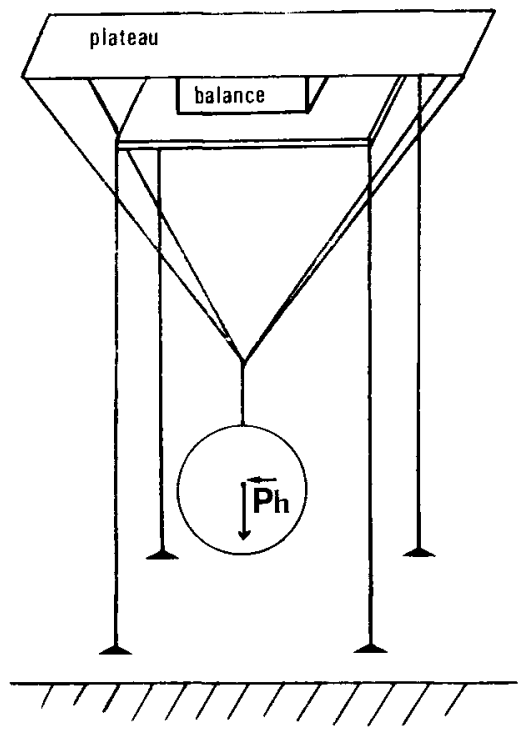

FIG. 3

Système de pesée de la rondelle retirée de l'eau.

Weighing method of the emerged sample.

Le chronomètre est déclenché au moment où la rondelle est sortie de l'eau, c'està-dire après $4 \mathrm{mn}$ d'immersion. Les lectures sont faites toutes les 5 secondes, elles ont été arrêtées à $4 \mathrm{mn}$ en raison de la stabilisation des valeurs observées.

Les 9 courbes obtenues sont semblables et un exemple en est donné en figure 4. 


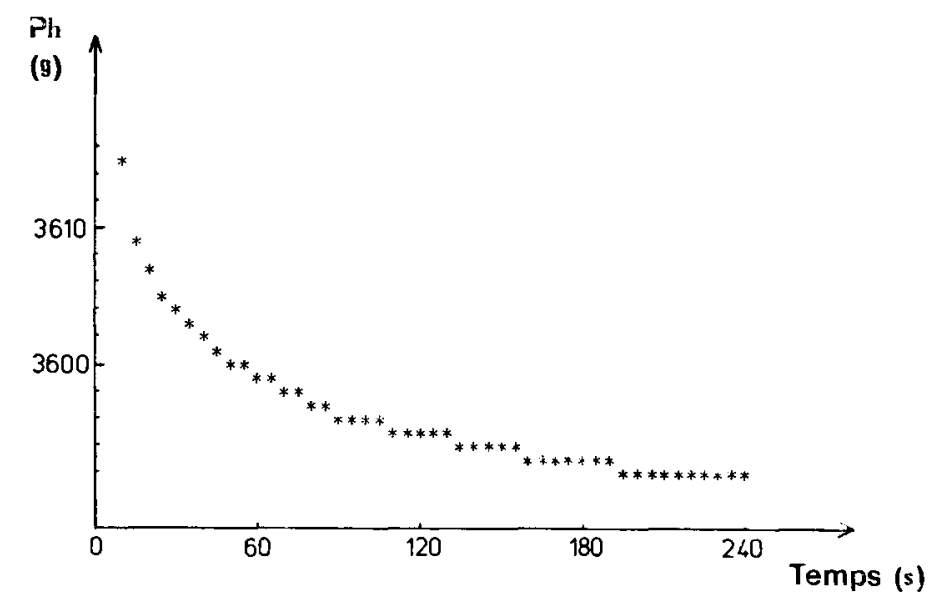

FIG. 4

Influence de la durée d'émersion sur le poids de la rondelle $n^{*} 9$. Influence of emergence time on weight of sample $n^{\prime \prime} 9$.

Nous interprétons leur allure en supposant que la décroissance assez rapide dans un premier temps est liée au départ de l'eau de surface qui circule sur la rondelle (l'écoulement est continu). Puis ensuite, l'eau pénétrée commence à sortir de la rondelle (l'eau s'écoule au goutte à goutte).

C'est à partir de cette interprétation simple que nous avons admis que l'eau absorbée pendant $4 \mathrm{mn}$ d'immersion était l'eau que la rondelle n'avait pas restituée $3 \mathrm{mn}$ après l'émersion. donc :

De la lecture du poids humide de la rondelle à $3 \mathrm{mn}$ (noté $\mathrm{Ph}(3 \mathrm{mn})$ ), on déduit

$$
\mathrm{V}_{\mathrm{o}} \mathrm{g}_{0} \mathrm{~g}=\mathrm{Ph}(3 \mathrm{mn})-\mathrm{P}_{\mathrm{f}}
$$

I1 découle alors de (3) et (5) :

$$
V_{\mathrm{f}} \varrho_{0} g=\mathrm{Ph}(3 \mathrm{mn})-\mathrm{P}_{\mathrm{ap} 1}
$$

Nous avons calculé, pour chaque rondelle, l'erreur relative sur $V_{f}$ que l'on fait en choisissant $\mathrm{Ph}(3 \mathrm{mn})$ pour le calcul, si l'on avait dû choisir en réalité $\mathrm{Ph}(1 \mathrm{mn})$, $\mathrm{Ph}(2 \mathrm{mn})$ ou $\mathrm{Ph}(4 \mathrm{mn})$. Pour la rondelle numéro $\mathrm{k}$ et pour le temps $\mathrm{i}(\mathrm{i}=1,2$ ou $4 \mathrm{mn}$ ), l'erreur est définie par :

$$
\mathrm{er}_{\mathrm{k}}(\mathrm{i})=\frac{\mathrm{V}_{\mathrm{fk}(\mathrm{i})}-\mathrm{V}_{\mathrm{fl}(3)}}{\mathrm{V}_{\mathrm{fk}(\mathrm{i})}}
$$

avec $: V_{\mathrm{fk}}(\mathrm{i})=P h(\mathrm{i} m n)-P_{\mathrm{ap},}$,

Le tableau 2 fournit l'erreur moyenne et son écart-type estimé avec les 9 rondelles pour chacun des temps i. 
Leur valeur peut justifier a posteriori le choix de $\mathrm{Ph}(3 \mathrm{mn})$ comme poids humide de référence pour le calcul de $\mathrm{V}_{\mathrm{f}}$ : si le poids humide que l'on doit prendre en compte pour le calcul de $V_{f}$ se situe bien entre 1 et $4 \mathrm{mn}$ après l'émersion, l'erreur relative maximale obtenue sur $V_{f}$ en le calculant avec $\mathrm{Ph}(3 \mathrm{mn})$ est de 0,3 p. 100.

Nous avons voulu nous assurer que l'estimation de $V_{\mathrm{f}}$ était indépendante du temps d'immersion.

Deux des 9 rondelles ont été laissées dans l'eau pendant $24 \mathrm{~h}$. On a alors noté leur poids apparent dans l'eau et leır poids humide $3 \mathrm{mn}$ après leur émersion, puis on a calculé leur volume et les différences relatives avec les volumes estimés avec l'immersion de $4 \mathrm{mn}$.

Les résultats sont présentés dans le tableau 3.

\section{TABLEAU 2}

Moyenne et écart type des erreurs relatives sur $V_{j}$ estimé avec le poids humide des rondelles à $3 \mathrm{mn}$, s'il avait dî l'être avec le poids humide à 1,2 ou $4 \mathrm{mn}$.

Mean and standard-deviation of relative errors on $\boldsymbol{V}_{f}$ estimated with the fresh weight of samples after $3 \mathrm{mn}$ emersion, if calculated with the fresh weight after 1, 2 or $4 \mathrm{mn}$ of emersion.

\begin{tabular}{|c|c|c|}
\hline $\mathrm{i}$ & $m(i) \cdot 10^{3}$ & $\sigma(\mathrm{i}) \cdot 10^{3}$ \\
\hline 1 & 2,2 & 0,7 \\
\hline 2 & 0,8 & 0,2 \\
\hline 4 & 0,4 & 0,1 \\
\hline
\end{tabular}

$\mathrm{m}(\mathrm{i})=$ moyenne des $\mathrm{er}_{\mathrm{ki}}$ (i).

$\sigma(i)=$ écart type estimé sur l: moyenne.

$i=1,2$ ou $4, k$ variant de 1 à 9 .

\section{Tableau 3}

Comparaison des valeurs de $V_{f}$ obtentues pour 2 rondelles après $4 \mathrm{mn}$ et $24 \mathrm{~h}$ d'immersion.

Comparison between values of $V_{f}$

obtained for 2 samples after $4 \mathrm{mn}$ and 24 hours of immersion.

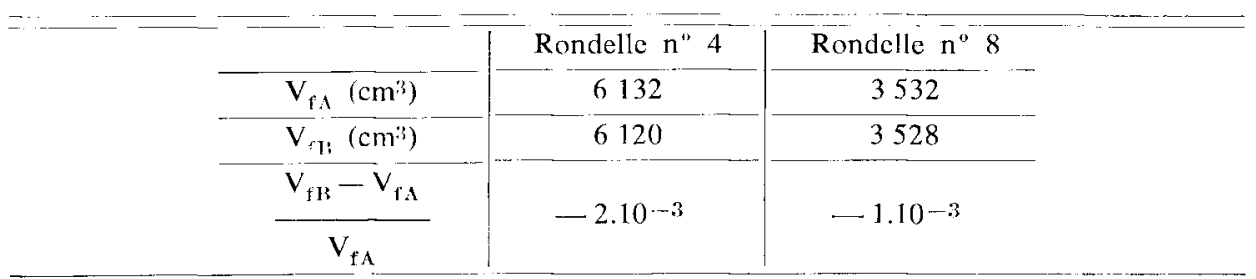

$\mathrm{V}_{\mathrm{fA}}$ : Volume estimé après $4 \mathrm{mn}$ d'immersion. Estimaled volume after $4 \mathrm{mn}$ of immersion.

$\mathrm{V}_{\mathrm{fH}}$ : Volume estimé après $24 \mathrm{~h}$ d'immersion. Estimated volume after 24 hours of immersion. 
Les faibles valeurs des différences obtenues indiquent bien que le résultat est indépendant du temps d'immersion ou encore du taux d'humidité initial de la rondelle en condition naturelle qui est toujours supérieur au point de saturation des fibres.

Pour un taux d'humidité inférieur, des déformations (gonflement dans l'eau) modifieraient les résultats (VALLı̀̀re, 1975).

\subsection{Relation entre $V_{o}$ et les dimensions de la rondelle}

La valeur des coefficients de corrélation linéaire entre le volume d'eau absorbé $V_{1}$ et les différentes surfaces d'échanges de la rondelle indique que l'écorce est perméable à l'eau dans le sens radial. Ces coefficients valent respectivement 0,061 pour la section transversale $\frac{\mathrm{C}^{2}}{4 \pi} ; 0,603$ pour la surface de l'écorce $\mathrm{C} . \mathrm{H}$ et 0,623 pour la surface totale.

De plus, le coefficient de corrélation entre $V_{0}$ et le volume de l'écorce calculé géométriquement par $\mathrm{V}_{\mathrm{c}}=\mathrm{C} . \mathrm{H} . \mathrm{e}-\pi . \mathrm{e}^{2} . \mathrm{H}$ est significatif au seuil d'erreur de 5 p. 100 et vaut 0,776 (fig. 5). (C, $\mathrm{H}$ et e sont les données du tableau 1).

C'est done l'écorce des rondelles qui absorbe la plus grande partie du volume d'eau $V_{w}$.

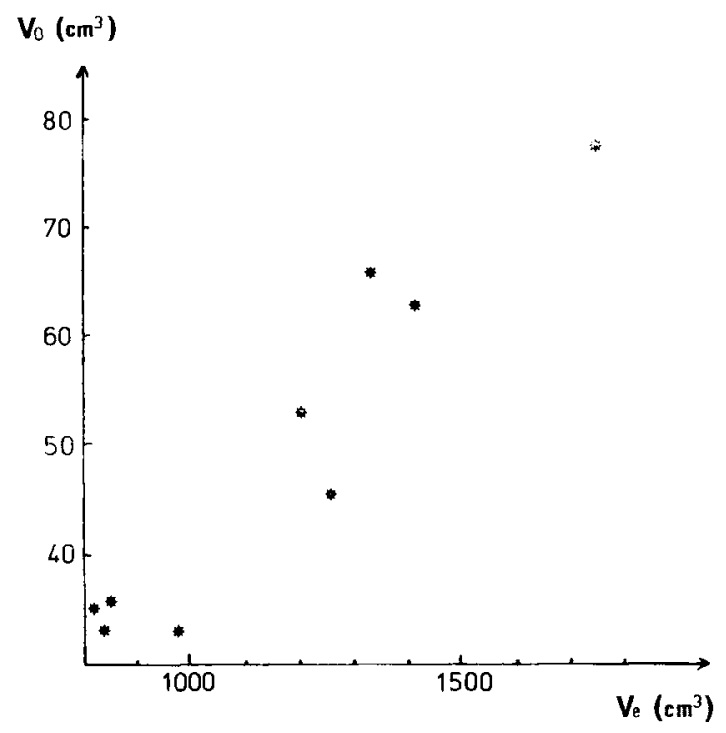

FIG. 5

Volume d'eau absorbé par la rondelle en fonction du volume de son écorce.

Volume of water absorbed by the sample in relation with bark volume. 


\section{Discussion}

Les calculs ont été établis avec le poids apparent des rondelles au bout de 4 minutes d'immersion. L'expérimentateur pourra réduire ce temps à 2 minutes sans altérer considérablement la précision des résultats. Pour les rondelles de l'expérience présentée ci-dessus, le poids apparent augmentait au plus de $3 \mathrm{~g}$ entre 2 et 4 minutes, soit une variation relative maximale par rapport au poids frais de 0,1 p. 100 .

Le nombre de rondelles avec lesquelles ont été effectuées les expériences est peu élevé. Il s'agissait non pas d'établir une équation à partir de résultats expérimentaux, mais de vérifier avec quelques données numériques l'exactitude d'une formule préalablement établie.

La formule (4) a été établie en admettant que les rondelles immergées dans l'eau pendant quelques minutes ne subissaient pas de déformations par rapport à leur état initial.

Cette hypothèse a été vérifiée pour le bois (Rosen, 1974; Vallière, 1975). Par contre, l'écorce subit certainement un léger gonflement; nous pouvons alors raisonnablement admettre que la variation de volume qu'il provoque est négligeable par rapport au volume total de l'échantillon.

Cet article est consacré à l'amélioration du calcul du volume d'un échantillon de bois par la méthode d'Archimède, appelée encore méthode hydrostatique par CARre (1972). Notons cependant qu'il sera également possible de corriger le calcul du volume obtenu par la méthode du xylomètre en tenant compte du volume d'eau absorbé pendant l'immersion. Il suffira d'effectuer la différence des poids de l'échantillon avant l'immersion et environ $3 \mathrm{mn}$ après qu'il soit retiré de la cuve.

Nous insistons sur le fait que les expériences fournissant quelques résultats chiffrés ont été réalisées avec des rondelles de chênes non écorcés. Pour une autre essence, à l'écorce moins poreuse (hêtre, charme), il est fort possible que le gain en précision obtenu en tenant compte du volume d'eau absorbé soit faible dans l'absolu et par rapport au travail que fournit une pesée supplémentaire (poids humide de l'échantillon). Il en est de même pour des échantillons écorcés, puisque l'essentiel de l'eau semble être absorbé par l'écorce.

D'autres facteurs peuvent contrôler la quantité d'eau absorbée pendant l'immersion : la porosité du bois, sa densité, son taux d'humidité, la forme et les dimensions de l'échantillon, l'épaisseur de la colonne d'eau qui le surmonte...

Nous pensons notamment que l'erreur relative réalisée en négligeant le volume d'eau absorbé sera d'autant plus grande que le rapport de la surface totale de l'échantillon à son poids frais sera plus élevé. Ainsi, sur des carottes prélevées à la tarière, l'erreur sera probablement très importante.

Cette hypothèse ne s'avère pas exacte pour notre échantillon de 9 rondelles : la corrélation linéaire entre l'erreur et le rapport surface totale/poids frais est très mauvaise. Nous supposons que les variations dimensionnelles des rondelles sont insuffisantes; de plus, le taux d'humidité est pratiquement constant d'un échantillon à l'autre. 


\section{Conclusion}

Nous avons démontré que l'utilisation de l'équation :

$$
V_{\mathrm{f}} Q_{o} g=P_{f}-P_{a p p}
$$

pour calculer le volume d'un échantillon de bois à l'état frais par la méthode hydrostatique (ou méthode d'Archimède) n'était pas juste.

En théorie, il faut tenir compte du volume d'eau absorbé par l'échantillon pendant son immersion.

L'équation exacte que l'on doit utiliser est :

$$
\mathrm{V}_{\mathrm{f}} \varrho_{0}, \mathrm{~g}=\mathrm{P}_{\mathrm{h}}-\mathrm{P}_{\mathrm{a} \mathrm{pp}}
$$

où $\mathrm{P}_{\mathrm{app}}$ est le poids apparent de l'échantillon dans l'eau juste avant qu'il ne soit retiré de la cuve et $P_{h}$ son poids humide $3 \mathrm{mn}$ après son retrait de la cuve.

L'utilisation de (I) au lieu de (II) entraîne une sous-estimation du volume calculé et par conséquent une surestimation du taux de conversion du volume en biomasse et de la biomasse estimée.

En pratique, nous avons constaté que la sous-estimation pour des rondelles de chêne non écorcées, dont le poids à l'état frais variait entre 3 et $8 \mathrm{~kg}$ était en moyenne de 1,2 p. 100 avec un écart-type par rapport à cette moyenne de 0,5 p. 100.

L'emploi de l'équation (II) supprime donc un biais systématique de + 1,2 p. 100 sur la biomasse estimée. L'erreur maximale sur le volume de l'échantillon est estimée à 0,3 p. 100.

L'erreur sur la pesée à l'état sec des échantillons étant du même ordre de grandeur dans les cas les moins favorables, c'est-à-dire pour des échantillons de poids sec voisin de $300 \mathrm{~g}$ pesés avec une balance dont la précision est le gramme ; le taux de conversion de volumes en biomasse défini par le rapport du poids anbydre d'un échantillon à son volume frais est obtenu avec une erreur relative maximale de 0,6 p. 100.

Mais attention, cette précision sur le taux de conversion ne doit pas leurrer l'utilisateur ; l'erreur sur le volume de la grume ou des billons dont il veut connaître la valeur en biomasse est nettement supérieure à 0,6 p. 100.

Le taux de conversion est calculé avec un volume réel alors que le volume des billons est un volume géométrique.

La seule prétention de l'auteur est de supprimer un biais systématique dans le calcul d'un terme de conversion de volumes en biomasse couramment utilisé par le praticien dans ce domaine.

Reçu le 5 août 1985.

Accepté le 6 février 1986. 


\section{Summary \\ The conversion factor of fresh wood volumes into biomass : improving its methods of estimation}

A study of the literature shows that the Archimedean principle is frequently incorrectly used to determine the volume of a fresh or oven-dry sample of wood.

The fact that the quantity of water absorbed by a sample during the immersion is not taken into account, produces a systematical underestimation of the volume. The conversion factor of volume into biomass, defined by the ratio of oven-dry weight of the sample to its fresh volume is then overestimated, as also is the biomass, estimated by using this conversion factor.

An equation giving an exact expression of the volume is established with a theoretical demonstration. We then experimentally test it with non de-barked samples of oak (Quercus petraea Liebl. or Quercus robur L.) of which the ratio of moisture to fresh weight is about 45 p. 100.

For these samples, we suppress a mean systematical bias of 1.2 p. 100 and the conversion factor is determined with a relative maximum error of $0.6 \mathrm{p} .100$.

Also, we show that a linear correlation exists between the volume of water absorbed by the sample and the bark volume.

It is stressed that the increase in precision certainly depends on the sample : dimensions, form, wood porosity, presence or absence of bark, etc. method.

The volume of absorbed water must also be considered when one uses the xylometer

Key words : Biomass, methods of estimation, sample, volume, precision, Quercus.

\section{Références bibliographiques}

ALEMDAG I.S., 1982. Methods of estimating forest biomass from stand volumes : A case study with Ontario Jack Pine. Pulp Paper Canada, 83, 41-43.

Auclair D., Metayer S., 1980. Méthodologie de l'évaluation de la biomasse aérienne sur pied et de la production en biomasse des taillis. Acta Ecologica, Ecol. Appl., 1, 357-377.

Auclair D., Bige M.C., 1984. Une méthode d'évaluation régionale de la biomasse des taillis à partir des données de l'Inventaire Forestier National. Application à la région Centre. Ann. Sci. For., 41 (4), 405-426.

Bouchon J., Nys C., Ranger J., 1985. Cubage, biomasse et minéralomasse : comparaison de trois taillis simples des Ardennes Primaires. Acta Ecologica, Ecol. Plant., Vol. 6 (20), $\mathrm{n}^{\circ} 1,53-72$.

CARrÉ J., 1972. Evolution des caractéristiques de bois d'industrie en fonction de leur diamètre. Minist. de l'Agric., Station de Technologie forestière de Gembloux. Rapport d'activité, 9-31.

Kestemont P., 1970. Etude de la biomasse et de la productivité de trois peuplements en taillis simple. Bull. Inst. r. Sci. nat. Belg., 46, 17.

Kestemont P., 1971. Biomasse et productivité aériennes d'un taillis de chênes et bouleaux riche en stellaires et violettes du Bois de Rope (Orchimont). Bull. Soc. r. Bot. Belg., 104 (1), 91-102.

LEGUAY F., 1982. Etude de la répartition de la biomasse dans les réserves de taillis sous futaie de chênes. INRA, Station de Recherches sur la Forêt et I'Environnement, doc. $82 / 36,81$ p. + annexes. 
MaRTin A.J., 1984. Testing volume equation accuracy with water displacement techniques. For. Sci., 30 (1), 41-50.

PARdÉ J., 1977. Biomasses forestières et utilisation totale des arbres. Rev. For. Fr., XXIX (5), $333-342$.

Ranger J., Nys C., Ranger D., 1981 a. Etude comparative de deux écosystèmes forestiers feuillu et résineux des Ardennes primaires françaises. I. Biomasse aérienne du taillis sous futaie. Ann. Sci. For., 38 (2), 259-282.

Ranger D., Nys C., Ranger J., $198 \mathrm{I}$ b. Etude comparative de deux écosystèmes forestiers feuillu et résineux des Ardennes primaires françaises. II. Biomasse aérienne d'une plantation équienne d'Epicea commun (Picea abies Karst). Ann. Sci. For., 38 (3), 377-388.

Rosen H.N., 1974. Penctration of water into hardwoods. Wood and Fiber, 5 (4), 276-287.

SchNock G., 1983. Volume, biomasses, surfaces d'échange et autres caractéristiques dendrométriques des tiges de charme (Carpinus betulus L.). Acta Ecologica, Ecol. Applic., Vol. 4, $\mathrm{n}^{\circ} 4,325-342$.

VALLIÈre A., 1975. Moyens pratiques pour obtenir quelques caractéristiques des bois métropolitains. Bull. Inf. Tech., C.T.B., 74, p. 6-14. 